\title{
Considerations for Successful Scale-Up To Tox Batches And Phase-Api (Bulk Drugsubstance)
}

\author{
Krishna Sarma Pathy* \\ Head /QC/QAIPL research centre, Department of Pharmacy, India
}

Received: 䟧 August 17, 2018; Published: 䟧 August 28, 2018

*Corresponding author: Krishnasarma pathy, Head /QC/QAIPL research centre, Department of Pharmacy, India

\begin{abstract}
Practical Considerations in Pharmaceutical Production Scale-Up- This paper is meant to provide a high-level overview of the steps required to prepare the bulk drug substance API. From first-in-human and through each of the clinical trial stages and into commercialization, scale-up of formulation design is a natural part of pharmaceutical development. At each stage of process development, the batch size increases, from laboratory-scale batches that may be quite small to support preclinical and early clinical stages, to pilot batches that are used in process development, and finally to the production-scale batches needed to support commercialization.
\end{abstract}

\section{Introduction}

Since phase 1 studies are generally first in human studies, they are the initial baseline for establishing the safety of the product in people. Because changes to product quality could affect patient safety, this paper focused on establishing a controlled manufacturing process and a set of analytical assays to detect any changes to the product which could potentially impact patient safety. In addition, a specific set of studies have also been discussed that also help to ensure patient safety during the phase 1 clinical trial. If the points in the paper are followed, the elements for a strong IND bulk drug substance development package and robust manufacturing process should be in place. In the beginning, we don't pay too much attention to yields as long as the conversion is relatively decent. Before we start doing experiments, we focus first on things like which reagents to use and how to make the process as efficient as possible. We see what tricks we can use that we learned from other projects. Efficiency is the key. The more efficient the chemistry, the easier to purify and to meet ICH guidelines.

As the life cycle of drug development unfolds, the demands on the synthetic process will change. In early development, the emphasis is very much on timely delivery of bulk supplies of the API using a safe process. Thus, most of the SELECT criteria can usually be satisfied when preparing the first few kilograms of the API or New Chemical entity (NCE) in bulk. In these early clinical trial stages, the most frequent issue encountered involves patient safety. However,

we are also focusing our efforts on attaining the highest yield, the lowest number of impurities, the easiest purification process, the least amount of pressure and most moderate temperature. In other words - the most efficient process. By the time that a drug candidate reaches Phase III clinical trials, the CMO will need to manufacture perhaps hundreds of kilograms of API and the demands on the process become more acute across the full range of SELECT criteria.

The most important factors in developing "right-first-time" processes
a) Chemical yield
b) Cycle time
c) Number of chemical steps and convergence
d) Use of higher molecular weight protecting group and reagents

e) Number of energy-consuming operations.

Implementing this approach is key to reducing API development time as complexity grows and budgets shrink. As with any risk management plan, the goal is to be proactive in finding and mitigating sources of risk. This is accomplished by removing unwanted variability in each stage of a process. In clinical trials, the emphasis is on identifying, reducing, and monitoring risks to 
patient safety, data integrity, regulatory and protocol compliance, and project scope (both budget and timelines). Paying close attention to these five variables above yields the most advantageous synthetic route. Material for toxicology studies and Phase 1 clinical investigations is often prepared from the same batch. One benefit of preparing only one batch is that the labor charges are less than they would be for two batches, and in early phases of drug development the cost of labor is much greater than the cost of raw materials. A second benefit is that the impurity profile of material first going into man will be that of the material used for the tox batches; this is significant, because the tox studies qualify the impurities, and subsequent active pharmaceutical ingredient (API) batches for human use must have no new impurities, only impurities with levels no greater than those found in the tox batches [1-15].

A disadvantage of preparing a large amount of material for both tox and Phase 1 is that the excess material will be wasted if the drug candidate fails during the tox studies. For tox and Phase 1 batches it is essential to set reasonable goals for API purity and use unit operations that would likely be employed on scale. A purity of $98 \%$ is reasonable; the FDA will readily permit companies to upgrade the routine quality of their API specifications after anticipated optimization, but a request to downgrade the drug substance purity specification will likely not be encouraged. As a worst-case scenario, suppose that a purity of $99.8 \%$ could not be met because scrutiny of a new HPLC method showed that a highly purified reference standard was only $99.0 \%$ pure. Under such circumstances additional steps, such as treatment with activated carbon or recrystallization, may be necessary, increasing the overall cost of goods (COG).

\section{Challenging the Parameters}

Table 1.
In order to prepare material of $98 \%$ purity for the tox batches, it is necessary to understand and control the isolation of the drug candidate. Once optimal isolation conditions have been identified, the batch may be isolated under slightly less than optimal conditions, for example, by cooling the crystallization slurry rapidly or by applying smaller volumes of washes to the wet cake on the filter. Another approach is to crystallize the product in the presence of additional typical process impurities; for instance, the product may be crystallized in the presence of impurities added as a portion of the mother liquor from a prior batch. Other impurities that need to be controlled in tox batches and subsequent batches include metals, such as palladium, Class 1 and Class 2 residual solvents (www.fda.gov), and any by-products known to be toxic.

[Note that, in the passive approach to removing palladium residues, a step using $\mathrm{Pd}$ is positioned early in a route and the amount of Pd in process streams is reduced by attrition in the workups of successive steps. A number of approaches are avail- able to actively remove $\mathrm{Pd}$, and these have been recently reviewed. Not surprisingly, the size, speed and power of the equipment involved in production has a significant impact on the entire process and must be matched to the batch-size requirements for production efficiency. Regardless of the size of the batch and its applicationfor use in clinical trials and stability testing, to service a smaller patient population with an orphan drug, or to provide for a larger population with a commercial product - the object is always the same: the finished product must be identical to the original formulation design regardless of the volume under which it is was produced.

\begin{tabular}{|c|c|c|c|c|}
\hline Attribute & Proposed acceptance criteria & Release testing & Internal testing" & Stability testing \\
\hline Description & $\begin{array}{l}\text { Range of color description (e.g., white to almost- } \\
\text { white to light yellow powder) }\end{array}$ & $x$ & - & $x$ \\
\hline Identification by spectroscopic method & Spectrum conforms to that of reference & $x$ & - & - \\
\hline Counterion & Report results & $x$ & $\mathrm{x}$ & - \\
\hline Assay & $\begin{array}{l}\text { 97.0-103.0\% "anhydrous basis" or "anhydrous and } \\
\text { solvent free basis" if compound is a solvate }\end{array}$ & $x$ & - & $x$ \\
\hline Impurities/Degradation products' & $\begin{array}{l}\text { Individual NMT } 1.0 \% \\
\text { Total NMT } 3.0 \%\end{array}$ & $\mathrm{x}$ & $x$ & $x$ \\
\hline Chiral impurity ${ }^{2}$ & NMT $1.0 \%$ & $\mathrm{x}$ & $\mathrm{x}$ & $\mathrm{x}$ \\
\hline Residual solvents $^{3}$ & $\begin{array}{l}\text { ICH limits or other justified limits for solvents used in } \\
\text { the final synthetic step }\end{array}$ & $x$ & $\mathrm{x}$ & - \\
\hline Mutagenic impurities & $\begin{array}{l}\text { Follow the referenced guidance (Ref. 13) until ICH M7 } \\
\text { is finalized }\end{array}$ & - & $\mathrm{x}$ & - \\
\hline Inorganic impurities & NMT EMA limits/ADI & - & $x$ & - \\
\hline Water content & Report results & - & $\mathrm{x}$ & $x$ \\
\hline Solid form ${ }^{4}$ & Report results & - & $x$ & $x$ \\
\hline Particle size & Report results & - & $\mathrm{x}$ & - \\
\hline ROI & NMT $1.0 \%$ & - & $\mathrm{x}$ & - \\
\hline \multicolumn{5}{|c|}{$\begin{array}{l}\text { 'In addition to the acceptance criteria, internal targets may be used to trigger action at the proposed } 3 \mathrm{X} \text { ICH identification }(0.3 \%) \text { or qualification }(0.5 \%) \text { limits. } \\
\text { Table } 2 \text { provides qualification scenarios for individual impurities based on levels in the initial lot used for GLP safety studies versus lots produced for Phase } 1 \\
\text { through Phase 2a clinical studies. }{ }^{2} \text { For a DS with two or more chiral centers, specific rotation may be used to monitor chiral purity in early development due } \\
\text { to the complexity of the molecule. Chiral impurities can also be monitored and/or controlled upstream. ' } \text {. Solvents used in earlier steps of the synthetic process } \\
\text { can be monitored as internal specifications. ' Physical properties, such as polymorphic form and particle-size distribution, are typically monitored throughout } \\
\text { development as non-specification characterization tests. As development progresses towards commercialization, specifications may be introduced. 'Internal } \\
\text { testing can be performed in addition or in replacement of release testing on the final DS. Internal testing may have target acceptance criteria tighter than the }\end{array}$} \\
\hline
\end{tabular}


Although some scale-ups are easier than others by virtue of their manufacturing process, scale-up is always a meticulous process of trial and error. For each stage of the scale-up process, the product must be physically and analytically tested against approved physical and analytical specifications and the critical process parameters must be challenged in order to ensure the process can be validated. For example, with a wet granulation in the high-shear granulator, we may begin the rate at which the granulation solution is applied to the blend at 1000 grams per minute and then compare that to a batch in which the same amount of granulation solution is added at 3000 grams per minute. If there's no change in the net result, a range is established for validation batches and we could improve process efficiency by adding granulation solution at three times the original rate. This type of process is carried out for every stage of production regardless of whether formula is being scaled up from two-kilo laboratory batches to 10 -kilo study batches or 1000-kilo production batches. The process is also repeated for any scale-up of more than 10 times the original size. To scale up a 10-kilo batch to 1000 kilos, the entire scale-up process would have to be repeated first when we scale from 10 to 100 kilos and again when we scale from 100 to 1000 kilos according FDA Scale-Up and Post-Approval Changes (SUPAC) guidelines (Table 1).

\section{Conclusion}

\section{Gaining Regulatory Approval}

As with all stages of pharmaceutical production, scale-up requires careful planning and meticulous documentation of data. Working with the client, the technical transfer team develops a process development protocol and prepares batch manufacturing instructions. As the final step, a process development report is prepared as part of the submission package. Comparing data from each step in the process helps determine scalability requirements and identify critical process parameters at full scale. When successful physical and analytical testing at each stage is complete, the first full-scale feasibility batch can be produced and fine-tuned to maximize process efficiencies. When the technical transfer team is satisfied with the process development or feasibility batches, registration batches are produced for submission to regulatory agencies. If approved, validation batches are produced in order to establish that the process is valid, stable and able to reliably reproduce the product formulation performance, efficacy and safety as originally devised. Ideally all subsequent batches will be prepared by the route and process used for tox and/or Phase 1 batches, so that on-scale impurities and impurity profiles will meet the guidelines above of course, it is difficult to predict the final optimized process for a drug candidate. The best approach to control impurities is to determine the optimal starting materials, reagents, process, and final form (salt, polymorph) early ("freeze" the final step)

\section{References}

1. Gordon Amidon HL (1995) A Theoretical Basis for a Biopharmaceutics Drug Classification: The Correlation of In Vitro Drug Product Dissolution and In Vivo Bioavailability. Pharmaceutical Research 12(3): 413-420.

2. (2017) United States Food and Drug Administration C (2000, August). Waiver of In Vivo Bioavailability and Bioequivalence Studies for Immediate Release solid Oral Dosage Forms Based on a Biopharmaceutics Classification System. Guidance for Industry. United States Department of Health and Human Services.

3. US Food and Drug Administration. (2008) CGMP for Phase 1 Investigational Drugs. Rockville, MD: U.S. Department of Health and Human Services.

4. Boudreau SP (2004, November) Method Validation by Phase of Development. Pharmaceutical Technology pp. 54-66

5. Gupta S, Saini S (2012) Technology Transfer in Pharmaceutical Industry An Overview. J Pharm Sci 2(3): 1-6.

6. Guidelines for Technology Transfer 2013.

7. Souder W, Nashar A, Padmanathan V (1990) Guide to The Best Technology Transfer Practice. J Technol Transf 1(2): 15-19.

8. Kaur A, Sharma O, Dhari J (2013) Technology Transfer in Pharmaceutical Industry. Int J Curr Pharm Res 5(1): 17-18.

9. Biruk A, Biruk Abate (2016) Technology transfer in pharmaceutical industries through product development and scale-up approaches: Challenges and opportunities for developing countries. IJETAE 6(7): 232-243.

10. (2007) Indian drug makers take to in-licensing for better health. IDMA Bulletin 18(17): 17-22.

11. www.moneycontrol.com/india/news/business/themis_aventistechransferdeal/16/07/294996.

12. Panltchpakdl S (2011) Local Production of Pharmaceutical and related Technology transfer in Developing countries. WHO 17(26): 52-70.

13. Ansher S, Gupta S, Wanger B (2003) National cancer Institute and Adriana Jenkins of Millennium Pharmaceutical 1(2): 364-421.

14. www.cdri.res.in Accessed on May 15, 2017

15. https://en.wikipedia.org/wiki/Tata_Institute_of_Funda Research. Accessed on 15 May 2017. mental 
(C) (P) This work is licensed under Creative

To Submit Your Article Click Here: Submit Article

DOI: 10.32474/LOJMS.2018.01.000112

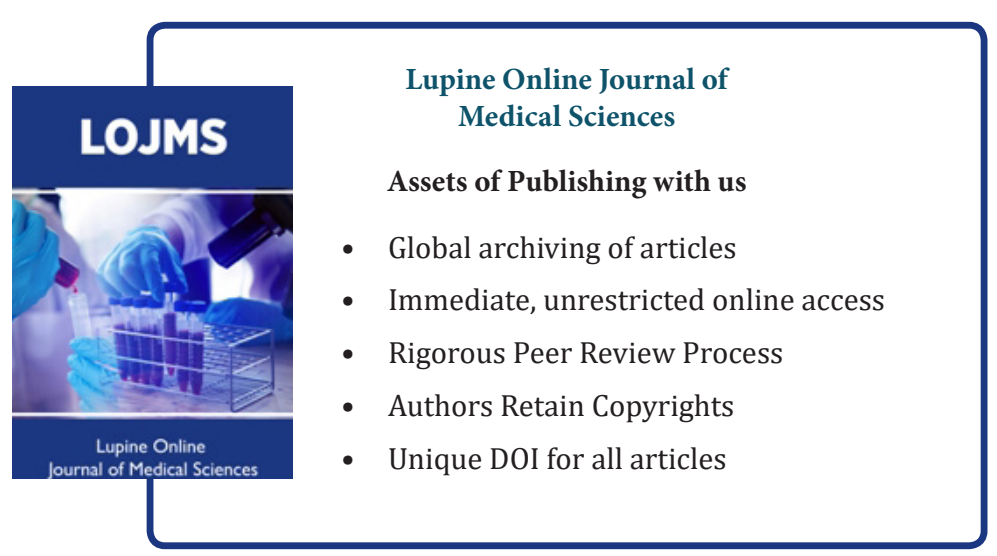

